\title{
The role of subvocal rehearsal in preschool children's prospective memory
}

\author{
Caitlin E.V. Mahy ${ }^{\mathrm{a}, *}$, Hannah Mohun $^{\mathrm{b}}$, Ulrich Müller ${ }^{\mathrm{b}}$, Louis J. Moses ${ }^{\mathrm{c}}$ \\ a Department of Psychology, Brock University, Canada \\ b Department of Psychology, University of Victoria, Canada \\ c Department of Psychology, University of Oregon, Eugene, OR, United States
}

\section{A R T I C L E I N F O}

\section{Article history:}

Received 6 October 2015

Received in revised form 6 June 2016

Accepted 8 July 2016

\section{Keywords:}

Prospective memory

Subvocal rehearsal

Verbal ability

Verbal working memory

Preschool children

\begin{abstract}
A B S T R A C T
The current study examined the impact of a verbal interference manipulation on 4- and 5year olds' prospective memory (PM). Children were randomly assigned to either complete a quiet delay activity (standard condition) or answer questions aloud during the delay activity (verbal interference condition). Children then completed a PM task followed by several individual differences measures (verbal working memory, inhibitory control, and receptive vocabulary). Four-year-olds showed worse PM than 5-year-olds, children in the verbal interference condition showed worse PM compared to the standard condition, and there was a marginal interaction between age and condition driven by poor performance of 4-year-olds in the verbal interference condition. PM performance was positively correlated with verbal working memory and receptive vocabulary (but not inhibitory control) in the verbal interference condition only suggesting that children with better verbal abilities were more able to cope with verbal interference to the benefit of their PM.
\end{abstract}

CC 2016 Elsevier Inc. All rights reserved.

Remembering to carry out a future intention, prospective memory (PM), has received increasing attention as an important aspect of young children's cognitive development (e.g., Kliegel \& Jäger, 2007; Kvavilashvili, Messer, \& Ebdon, 2001; Mahy \& Munakata, 2015). Age-related improvements in event-based PM during the preschool years have now been clearly established (Kliegel \& Jäger, 2007; Mahy \& Moses, 2011), and factors such as motivation, length of delay, and difficulty of the ongoing task have been found to affect young children's PM performance (for a recent review see Mahy, Moses, \& Kliegel, 2014).

A standard PM task involves two concurrent components: first, the prospective memory task itself-what children must do when a particular cue appears in the environment (e.g., a picture of an animal), and second, the ongoing task which acts as a distractor task in which the PM cues are embedded (e.g., a card sorting task). Prior to the onset of these two tasks, however, there is typically a delay period between the assignment of a PM intention and the start of the ongoing task. Despite the presence of a delay interval in virtually all PM tasks, little attention has been paid to processes occurring during this period that might affect later PM performance.

In one of the few studies examining characteristics of the delay period, Mahy and Moses (2011) found that the length of the delay interval has a significant impact on preschoolers' PM performance such that 4-year-olds' PM was not affected by delay length, but 5 -year-olds' PM actually increased after a long delay ( $5 \mathrm{~min}$ ) compared to a short delay ( $1 \mathrm{~min}$ ). The interpretation of these findings was that 5-year-olds may be refreshing their intentions during the delay and hence might benefit from a longer delay period due to increased opportunities to think and reflect on their future intentions. Further,

\footnotetext{
* Corresponding author at: Department of Psychology, Brock University, 1812 Sir Isaac Brock Way, St Catharines, ON L2S 3A1, Canada.

E-mail address: caitlin.mahy@brocku.ca (C.E.V. Mahy).
} 
Mahy and Moses (2015) found that filling the delay interval with a difficult visual working memory task resulted in worse later PM performance than when the delay was filled with an easy visual working memory task. They also found that children's executive abilities, including planning and working memory, were related to PM performance for children who received the difficult filler task but not for those who received the easy filler task. Taken together, these studies suggest that important cognitive processes operate during the delay interval that have a significant impact on later PM, and that individual differences in executive control have an impact on children's PM under different experimental conditions.

Mahy and Moses $(2011,2015)$ have argued that children might take advantage of the delay period to refresh or monitor their intentions (hence, why longer intervals are helpful for older children's PM and why difficult delay tasks might disrupt these processes resulting in worse PM performance). One way in which this refreshing process could be affected is by disrupting subvocal rehearsal during the delay.

There are important theoretical reasons to examine the role of subvocal rehearsal in how children maintain a prospective intention. In his classic model of working memory, Baddeley (1992) suggested that working memory is composed of a central executive, a visuospatial sketchpad, an episodic buffer, and the phonological loop. For our purposes, an important aspect of working memory is the phonological loop, which allows for storage and rehearsal of speech based information and directly supports subvocal rehearsal of the contents of working memory. Importantly, the central executive does not have storage capacity so relies on the episodic buffer to maintain information over time. According to this model, information such as intentions can be stored in working memory via the episodic buffer but also can be refreshed or rehearsed directly through subvocal rehearsal in the phonological loop. It is probable, however, that an intention is not held in the focus of working memory consistently (as this would index a vigilance process rather than PM, see Brandimonte, Ferrante, Feresin, and Delbello, 2001) but rather that it fades in and out of the focus of attention during the delay period (Cowan, 1995, 2005). It would be unlikely that children would use a vigilance strategy during a PM task given that they are engaged in an ongoing task that is occupying their attentional resources.

Similarly, Vygotsky (1934/1986) emphasized the important role of children's inner speech for guiding their actions. He hypothesized that children first use speech interpersonally for communication but as development progresses speech is internalized and used to direct children's behavior (in the form of inner speech). This self-directed speech supports higher cognitive functions and allows children to plan ahead and think about solutions in advance. Taken together, there are important theoretical reasons to examine the role of subvocal rehearsal and private speech in children's maintenance of their prospective intentions over time, as this may be one process through which prospective intentions are refreshed and maintained over a delay period. Importantly, Vygotsky's work has not been explored in relation to children's PM and thus is an important as well as novel aspect of the current study.

Several lines of empirical work support the idea that subvocal rehearsal plays an increasingly important role in problem solving in early childhood (see Müller \& Kerns, 2015). First, consistent with Vygotsky's theory, the use of covert (private) task-relevant speech is positively associated with performance on planning tasks in 4-8-year-old (Al-Namlah, Fernyhough, \& Meins, 2006; Fernyhough \& Fradley, 2005). Second, during the preschool period children are more likely to label stimuli or use private speech when task demands increase (e.g., Fernyhough and Fradley, 2005; Müller, Zelazo, Hood, Leone, \& Rohrer, 2004) suggesting that private speech is particularly useful under higher cognitive demand. Finally, the function of verbal labels changes during the preschool period from being attention-directing to stimulating a richer conceptualization of the task at hand (Jacques \& Marcovitch, 2010). Consistent with Vygotsky's suggestion that overt (audible) private speech is gradually replaced by covert (inaudible) private speech, it has been found that the use of covert private speech linearly increases between 4- and 8-years of age, and that the use of overt private speech decreases during the same period (e.g., AlNamlah et al., 2006; Winsler \& Naglieri, 2003). Further, important changes in children's private speech between 3 and 5 years of age seem to indicate a progressive internalization of private speech (Winsler, de Leon, Wallace, Carlton, \& Willson-Quayle, 2003).

More directly relevant to the role of subvocal rehearsal in prospective memory, recent research has documented that children's capacity for private speech is linked to their performance on memory and planning tasks. For example, Al-Namlah, Meins, and Fernyhough (2012) showed that 4-7-year-olds' use of self-regulatory private speech was positively related to longer autobiographical narratives that included more specific memories and demonstrated greater narrative cohesion. Further, Lidstone and colleagues showed that articulatory suppression (i.e., disruption of subvocal rehearsal by constant repetition of the word "Monday") negatively affected 7-10-year-old children's performance on the Tower of London task (Lidstone, Meins, \& Fernyhough, 2010). Individual differences in private speech production in 8-10-year-olds also positively predicted performance on the Tower of London, digit span task, and two measures of spatial intelligence (Lidstone, Meins, \& Fernyhough, 2011). In sum, this literature suggests private speech is positively associated with memory performance and planning in early to middle childhood.

Given that subvocal rehearsal seems a likely candidate mechanism by which children refresh their prospective intentions, we set out to design an experiment that would disrupt subvocal rehearsal during the delay period. Thus, we designed a delay task that would interrupt any rehearsal or refreshing of intentions at regular intervals: Children were asked to draw pictures but every 20 s they were interrupted with a question they had to answer (e.g., what's your favorite color? how many brothers or sisters do you have? etc.). In contrast, in the standard condition, children were simply asked to draw pictures quietly so their subvocal rehearsal was not interrupted in any way by the experimenter.

In addition to this manipulation, children's verbal working memory (backward word span), inhibitory control (Grass/Snow and Head-Shoulders-Knees-Toes), and receptive vocabulary (Peabody Picture Vocabulary Test) were measured. We predicted 
that children who had higher verbal ability and verbal working memory (but not inhibitory control) would cope with the verbal interference condition better (higher PM performance) than children with lower verbal ability and verbal working memory, whereas these verbal abilities would not be as strongly related to PM of children in the standard condition. These predictions were based on the assumption that the verbal interference condition would disrupt rehearsal of the PM intention in the phonological loop leading to poorer PM performance. Thus, children with superior verbal and verbal working memory skills should better cope with this disruption to subvocal rehearsal compared to children with weaker verbal abilities in the verbal interference condition. In the standard condition, where subvocal rehearsal is not disrupted, verbal abilities should be less strongly related to PM performance. Further, inhibitory control should not impact children's ability to cope with verbal interference (because inhibition relates only weakly to verbal abilities; see Müller, Jacques, Brocki, and Zelazo, 2009) and should therefore not be strongly associated with PM performance in either experimental condition.

Taken together, theoretical models of subvocal rehearsal and empirical findings in the developmental PM literature offer motivation to investigate the role of subvocal rehearsal in the maintenance of young children's prospective intentions. Given that subvocal rehearsal slowly emerges with age (e.g., Al-Namlah et al., 2006; Winsler \& Naglieri, 2003), we chose the early preschool period to investigate its relation to PM. Past work suggests that there are marked changes in PM between 4and 5-years-old (e.g., Mahy \& Moses, 2011, 2015) that are possibly driven by age-related changes in self-regulation (e.g., Carlson, 2005; Espy, 1997; Hongwanishkul, Happaney, Lee, \& Zelazo, 2005) and social understanding (e.g., ; Wellman \& Liu, 2004; Wellman, Cross, \& Watson, 2001), as well as under-explored factors such as subvocal rehearsal and verbal ability. The current study sought to: (1) replicate the findings of age-related increases in PM between 4- and 5-year-olds, (2) examine the impact of a verbal interference condition on PM that would disrupt children from subvocal rehearsal of their intentions during the delay period, and (3) examine how verbal working memory, inhibitory control, and verbal abilities would relate to PM in the verbal interference condition compared to the standard condition.

\section{Method}

\subsection{Participants}

Seventy 4- and 5-year old children participated in the study. Data from five children could not be used because they failed to meet a criterion for inclusion in the PM task and one child's data could not be used due to experimenter error. The final sample consisted of 324 -year-olds ( 17 boys; $M=52.78$ months, $S D=3.16$ ) and 325 -year-olds ( 19 boys; $M=66.06$ months, $S D=4.16$ ). Children were mostly Caucasian and middle class consistent with the populations from which the samples were drawn. Participants were recruited from university participant databases and local preschools at the University of Victoria, University of Oregon, and Brock University.

\subsection{Procedure}

An experimenter interviewed children individually in the laboratory or in a quiet area in a daycare center in a single session that took approximately $45 \mathrm{~min}$. All tasks were administered in a fixed order, as is convention in individual differences research: PM task, Backward Word Span, Grass/Snow, Head-Shoulders-Knees-Toes, and PPVT-IV. Parents were asked to provide demographic information prior to their child participating in the study. At the end of the session, children were given a small toy and were thanked for their participation. The university ethics review boards at University of Victoria, University of Oregon, and Brock University approved all procedures.

\subsection{Measures}

\subsubsection{Prospective memory task (adapted from Mahy \& Moses, 2011)}

The PM task consisted of a card-sorting game requiring children to (a) tell Morris the Mole, a stuffed animal with poor daytime vision, where a red dot sticker was on each card: above or below the picture on the card (the ongoing task), and (b) make a novel response to cards with animals on them (the PM intention). Cards were $3 \times 3$ in. color pictures of everyday objects (e.g., food, furniture, toys). In contrast, target cards pictured animals. In addition to finding the dot on each card and telling Morris aloud whether it was on top or on the bottom, children were told that Morris was afraid of animals and that if they saw an animal card, they should hide it from Morris by placing it in a box approximately three feet behind them. To ensure children understood the ongoing task, they were asked to tell Morris where the red dot was on three cards and were corrected by the experimenter if they got any incorrect.

Once children were familiar with the task and indicated they understood the rules, a 3-min retention interval began. Children were randomly assigned to one of two conditions: (1) a standard condition where they were asked to draw pictures quietly while Morris the Mole took a nap, or (2) a verbal interference condition where children were told that Morris would ask them questions about themselves while they were drawing pictures. In this condition, Morris asked children a question every $20 \mathrm{~s}$ from a standardized list of 8 questions (see Table 1 ). All children responded to each question.

After the retention interval, the card game began and children were asked to tell Morris where the red dot was on each card. There were 2 stacks that both contained 30 cards; of these, three were target cards (animals). Targets were the 9th, 
Table 1

Questions posed in the Verbal Interference Condition.

\begin{tabular}{lll}
\hline & Question & Time question was asked \\
\hline 1. & What is your name? & $20 \mathrm{~s}$ \\
2. & What is your favorite color? & $40 \mathrm{~s}$ \\
3. & How old are you? & $1 \mathrm{~min}$ \\
4. & What are you drawing a picture of? $20 \mathrm{~s}$ \\
5. & Do you have any brothers or sisters? & $1 \mathrm{~min}, 40 \mathrm{~s}$ \\
6. & What is your favorite game to play? & $2 \mathrm{~min}$ \\
7. & What is your favorite toy? & $2 \mathrm{~min}, 20 \mathrm{~s}$ \\
8. & Do you go to school or daycare? & $2 \mathrm{~min}, 40 \mathrm{~s}$ \\
\hline
\end{tabular}

18 th, and 25th cards in the first stack and 5th, 12th, and 28th cards in the second stack. Children were asked to draw pictures for 1-min between the two stacks.

After children completed the card game they were asked, "what were you supposed to do when you saw a picture of an animal?" This control question ensured that participants remembered the initial rule and that forgetting didn't arise from a retrospective memory failure for the task instructions. As noted earlier, five children (four 4-year-olds and one 5-year-old) were excluded from the final analysis for failing this control question or for experimenter error. Two of these children were from the standard condition and three were from the verbal interference condition suggesting that task condition did not contribute to rule failures. Children were given a PM score out of six based on the number of target cards that were correctly placed in the box.

\subsubsection{Backward word span (Wechsler, 1974)}

Children were introduced to a game where they had to repeat a series of words in a backward order. The experimenter first demonstrated a trial by explaining that if Morris the Mole, a stuffed toy, said "fruit, zoo" they should say "zoo, fruit". Then children were given a practice trial that they had to pass in order to move on. There were 2 trials for each sequence length (of 2-5-words) where children started with two words and continued until they made two errors in trials within the same sequence length (administration continued if they got at least one of the trials correct). Children were given a score of 0 if they were not able to complete the practice trial and were given 1 point for each sequence length in which they performed at least one trial correctly. Thus, children could score up to 4 if they were correct on at least one of the 5-item trials correct.

\subsubsection{Grass/snow (Carlson E Moses, 2001)}

Children were asked to report the color of snow and grass to the experimenter. All children responded correctly. The experimenter then introduced the rules to a 'silly opposites' game where they had to point to the white square when he or she said 'grass' and to the green square when he or she said 'snow'. Children were given two practice trials in which they had to point to the correct color square and then place their hands back on handprints in front of the color squares. Once children passed these two trials, fourteen additional trials were administered for a total of 16 trials. Children received a score out of 32 based on whether each trial was correct ( 2 points), self-corrected ( 1 point), or incorrect ( 0 points). Inter-rater reliability on $28 \%$ of the data was substantial (Cohen's kappa $=0.753$ ).

\subsubsection{Head-shoulders-knees-toes (Ponitz, McClelland, Matthews, E' Morrison, 2009)}

After a warm-up phase where children were asked to follow the experimenter's commands, children were asked to do the opposite of what the experimenter said: They were told to touch their head when the experimenter told them to touch their toes, and to touch their toes when the experimenter said touch your head. Children were given four practice trials and had to get at least 3 out of 4 trials correct to advance to the test trials. Then, 10 Head-Toes (HT) test trials were administered where children had to do the opposite of what the experimenter demonstrated. Children were given a score out of 20 on the HT trials based on whether they responded incorrectly ( 0 points), had to correct themselves ( 1 point), or responded correctly ( 2 points). After the HT trials, children were introduced to a new rule: to touch their shoulders when the experimenter said touch their knees and to touch their knees when the experimenter said touch their shoulders. Four practice trials were administered in which children had to demonstrate basic understanding of the rules by scoring at least 3 out of 4 . Then, children began the second phase where they completed 10 Head-Shoulders-Knees-Toes (HSKT) trials that included all four types of instructions: touch your head, touch your toes, touch your shoulders, and touch your knees. Children needed to respond by performing the opposite response and were given a score on these HSKT trials out of 20. Children's scores on the HT and HSKT trials were combined to yield a total score out of a possible 40 points. Only children who passed both practice phases were included in the data analyses $(\mathrm{N}=47)$. Inter-rater reliability on $28 \%$ of the data was substantial (Cohen's kappa=0.772).

\subsubsection{Peabody picture vocabulary test (PPVT-IV, Dunn \& Dunn, 2007)}

In this standard measure of receptive vocabulary, the experimenter read a word aloud and children were asked to point to the picture that matched the word. Administration was continued until children made at least 8 errors within a given set of 12 words. Raw PPVT scores were calculated based on children's performance. 
Table 2

Means and standard deviations for all tasks.

\begin{tabular}{|c|c|c|c|c|}
\hline \multirow[t]{2}{*}{ Task } & \multicolumn{2}{|l|}{ 4-year-olds } & \multicolumn{2}{|l|}{ 5-year-olds } \\
\hline & Standard Condition & $\begin{array}{l}\text { Verbal Interference } \\
\text { Condition }\end{array}$ & Standard Condition & $\begin{array}{l}\text { Verbal Interference } \\
\text { Condition }\end{array}$ \\
\hline Prospective Memory Task (0-6) & $3.75(2.46)$ & $1.44(2.13)$ & $3.88(2.22)$ & $3.69(2.24)$ \\
\hline Ongoing Task (Red Dot Location Task; 0-54) & $52.56(1.86)$ & $53.00(1.37)$ & $53.37(.96)$ & $53.06(1.65)$ \\
\hline Backwards Word Span (0-4) & $0.94(.77)$ & $0.25(.48)$ & $1.80(1.08)$ & $1.81(1.05)$ \\
\hline Grass/Snow (0-32) & $25.29(6.31)$ & $25.47(5.80)$ & $27.81(4.43)$ & $25.81(5.43)$ \\
\hline Head-Shoulders-Knees-Toes (0-40) & $24.08(11.09)$ & $21.45(8.82)$ & $29.93(8.45)$ & $27.27(9.55)$ \\
\hline Peabody Picture Vocabulary Test (Raw scores) & $97.00(20.77)$ & $89.06(20.70)$ & $113.50(21.65)$ & $116.88(9.76)$ \\
\hline
\end{tabular}

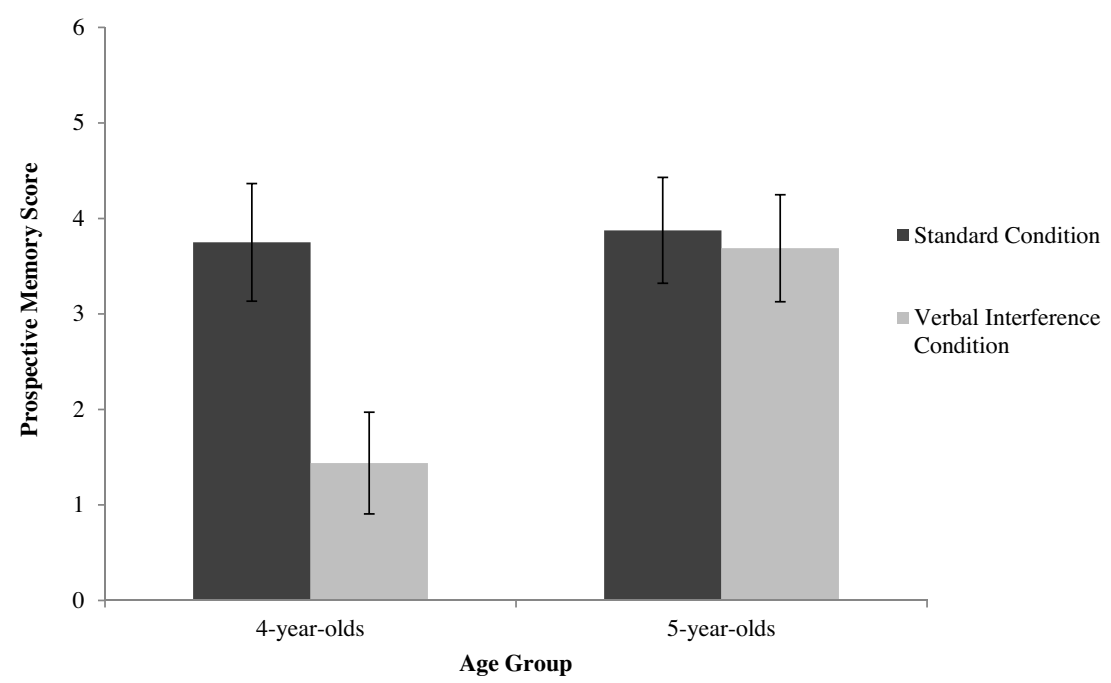

Fig. 1. Performance on the prospective memory task by age group and condition. Error bars represent 1 standard error.

\section{Results}

Table 2 shows the means and standard deviations of all measures for each age group. A 2 (Age Group: 4- vs. 5-year olds) by 2 (Condition: verbal interference vs. standard) ANOVA was conducted on PM performance. A significant main effect of age emerged, $F(1,60)=4.39, p=0.040, d=0.50$, with 5 -year-olds $(M=3.78, S D=2.20)$ outperforming 4 -year-olds $(M=2.59$, $S D=2.55)$. In addition, children in the verbal interference condition $(M=2.56, S D=2.44)$ had worse PM than children in the standard condition $(M=3.81, S D=2.31), F(1,60)=4.87, p=0.031, d=0.53$. Finally, there was a marginal interaction between age group and condition, $F(1,60)=3.52, p=0.066, \eta_{p}{ }^{2}=0.055$. Fig. 1 shows performance on the PM task by age group and condition. Simple effects revealed a significant difference between 4- and 5-year olds' PM performance in the verbal interference condition $(t=2.92, p=0.007, d=1.03)$ but no difference in the standard condition $(d=0.06)$. Four-year olds performed worse in the verbal interference condition compared to the standard condition $(t=2.85, p=0.008, d=1.00)$ but performance of 5-year-olds did not significantly differ between the conditions $(d=0.09)$.

Table 3 shows correlations between all measures and age. Children's age in months was positively correlated with performance on Backward Word Span, Head-Shoulders-Knees-Toes, and PPVT, $r$ s (57) $>0.332$, ps $<0.05$. Age in months was

Table 3

Correlations among all measures.

\begin{tabular}{|c|c|c|c|c|c|c|}
\hline & 1. & 2. & 3. & 4. & 5. & 6. \\
\hline 1. Age in Months & - & & & & & \\
\hline 2. PM Task & 0.203 & - & & & & \\
\hline 3. Ongoing Task & 0.190 & 0.057 & - & & & \\
\hline 4. Backward Word Span & $0.549^{* *}$ & $0.212^{\dagger}$ & $0.232^{\dagger}$ & - & & \\
\hline 5. Grass/Snow & 0.130 & 0.028 & 0.163 & $0.294^{*}$ & - & \\
\hline 6. Head-Shoulders-Knees-Toes & $0.262^{\dagger}$ & 0.131 & $0.326^{*}$ & 0.193 & $0.262^{\dagger}$ & - \\
\hline 7. Peabody Picture Vocabulary Test & $0.517^{* *}$ & $0.329^{* *}$ & 0.189 & $0.591^{* *}$ & 0.043 & 0.083 \\
\hline
\end{tabular}

$\dagger p<0.10$.

* $p<0.05$.

** $p<0.01$. 
Table 4

Correlations with PM by condition.

\begin{tabular}{lcc}
\hline Task & PM in standard condition & PM in verbal interference condition \\
\hline Backward Word Span & $-0.09(-0.05)$ & $0.41^{*}(.14)$ \\
Grass/Snow & $-0.08(.02)$ & $0.08(.04)$ \\
HSKT & $-0.01(-0.02)$ & $0.26(.19)$ \\
PPVT (raw scores) & $0.09(.06)$ & $0.56^{* *}(.35)$ \\
\hline
\end{tabular}

Note: Partial correlations controlling for age in months are in parentheses. HSKT = Head-Shoulders-Knees-Toes, PPVT= Peabody Picture Vocabulary Test. * $p<0.05$.

** $p<0.01$.

not related to PM performance, ongoing task performance, or Grass/Snow ( $p s>0.107$ ). PM and ongoing task performance were unrelated.

Table 4 shows raw and age-controlled correlations between the individual difference measures and PM performance by condition. In the standard condition, PM performance was uncorrelated with performance on any of the executive or verbal measures. In contrast, in the verbal interference condition, PM performance was significantly positively correlated with Backwards Word Span and PPVT score but not with measures of inhibitory control. Using z-tests, we found that the correlations between PM and Backwards Word Span and PM and PPVT score were significantly larger in the verbal interference condition compared to the standard condition, Fisher's $Z s>1.98, p s<0.049$. These correlations fell below significance, however, when age was controlled although the relation between PPVT and PM was still a medium sized effect.

We argue that poor PM performance of 4-year-olds in the verbal interference condition is due to the disruption of subvocal processes but an alternative possibility is that greater cognitive load as imposed by the demands of both drawing pictures and responding to pictures negatively affected their performance. In order to test this alternative, two regression analyses were performed predicting PM performance in both experimental conditions with age entered at the first step and backward word span (a working memory measure that would approximate children's ability to cope with increased cognitive load) and PPVT (a measure that would estimate their ability to cope with disruption to verbal processes) entered at the second step. In the verbal interference condition, age and PPVT were a significant predictors of PM performance, $B s>0.395$, $t$ s (31) $>2.27, p s<0.031$, whereas backward word span was not, $B=0.066, t(31)=0.281, p=0.781$. Age, backward word span, and PPVT were not significant predictors of PM performance in the standard condition ( $p s>0.45)$. These results suggest that our verbal interference manipulation was in fact targeting subvocal rehearsal (i.e., subvocal verbal processing) rather than just increasing cognitive load more generally, as verbal working memory was not a significant predictor of PM performance in the verbal interference condition where general verbal ability was.

\section{Discussion}

The goals of the current study were to: (1) examine age-related differences in PM between 4- and 5-year-olds, (2) examine the impact of an experimental manipulation on PM that would limit subvocal rehearsal of the intention during the delay interval, and (2) examine relations between PM performance, verbal working memory, inhibitory control, and verbal ability in the verbal interference condition compared to the standard condition. Findings revealed a main effect of age group and condition on PM performance with older children outperforming younger children, and children in the standard condition outperforming children in the verbal interference condition. A marginally significant interaction between age group and condition emerged and simple effects revealed that 4-year-olds' performance in the verbal interference condition was low compared to 5-year-olds' performance in that condition and 4-year-olds' performance in the standard condition. Verbal ability was a significant predictor of PM performance in the verbal interference condition whereas verbal working memory was not, suggesting that our manipulation successfully targeted subvocal rehearsal processes rather than manipulating cognitive load more generally. When correlations between PM and individual difference measures were compared separately by experimental condition, two different patterns emerged: there were no correlations between PM and the other measures in the standard condition; in contrast, there were positive correlations between PM and backward word span and PPVT scores in the verbal interference condition. These findings suggest that better verbal abilities are only related to better PM performance when there is verbal interference during the delay interval and not when there is no verbal interference during the delay interval. Interestingly, our measures of inhibitory control (Grass/Snow and HSKT) did not relate to PM in the verbal interference condition suggesting that it is verbal ability in particular, and not executive functions more generally, that relates to PM under conditions of verbal interference.

Given our results, it is clear that disrupting the opportunity for subvocal rehearsal during the delay period has a negative impact on children's PM. This is especially true for 4-year-olds, whose PM especially suffered under the verbal interference condition. It is possible that 4-year-olds still take advantage of the delay period to refresh their intention and that disrupting subvocal processing results in worse PM possibly due to the fact that their intention needs to be strengthened for optimal encoding. It is important to note, however, that although 4-year-olds' PM performance was poorer, these children could still produce the PM rule of what they were supposed to do, so it is unlikely that children had failed to encode the intention by the end of the PM task. In contrast, 5-year-olds were unaffected by the verbal interference condition suggesting that perhaps they rely less on subvocal rehearsal to refresh or maintain their intention or, alternatively, that the verbal interference condition 
tended to disrupt their subvocal rehearsal less than it did for 4-year-olds. For example, higher processing speeds might lead to more efficient refreshing of intentions in working memory (see Barrouillet, Bernardin, \& Camos, 2004), which might have contributed to the verbal interference condition having less of an impact on 5-year-old's PM performance compared to that of 4-year-olds due to age-related increases in processing speed. Our findings support the role of the phonological loop in maintaining a prospective intention in working memory (Baddeley, 1992) and the critical role that subvocal rehearsal/inner speech plays in directing one's own behavior, especially planning behavior (Vygotsky, 1934/1986).

The marginal interaction between age group and condition is somewhat surprising given that spontaneous subvocal rehearsal emerges with age. Thus it seems that older children should be more affected by a verbal interference manipulation. Similar to past research, however, it seems that younger children are more negatively affected by increases in difficulty during the delay whether executive (e.g., Mahy \& Moses, 2015) or linguistic in nature, as in the current study. Still, some alternative explanations for our results are worth considering. First, it is possible that rather than disrupting subvocal rehearsal our manipulation could have disrupted the latent verbal trace of the intention. This explanation seems unlikely given that all children could produce the intention at the end of the task without difficulty. Second, it is possible that the poorer performance of 4-year-olds in the verbal interference condition was due to the increased difficulty or increased engagement in the question-answer exchange in addition to drawing pictures (a general increase in cognitive load) compared to the standard condition and compared to 5 -year-olds in this condition. However, if our results were due to an increase in cognitive load more generally, we might expect that inhibitory control and verbal working memory would be important predictors of PM in the verbal interference condition as children with stronger executive abilities should be better equipped to cope with a greater cognitive demand. This was not the case; children's verbal ability was the only significant predictor of PM whereas inhibitory control and verbal working memory were not significant predictors of PM performance suggesting that the condition specifically targeted verbal processing rather than cognitive demand more generally. In sum, it seems that young children's subvocal rehearsal might be emerging and especially sensitive to interference at 4 years of age (e.g., Müller et al., 2004; Zelazo, 2004) whereas by 5-years children might be better able to cope with verbal interference and possibly recruit other strategies (such as visual representation) to refresh or maintain one's prospective intention.

In line with our prediction, results showed that children with better verbal abilities (measured by the backward word span and PPVT) coped with the verbal interference condition better than children with poorer verbal ability. This suggests that having better verbal skills is related to PM but only when the manipulation targets verbal processing (since there was no relation between verbal ability and PM in the standard condition). There is evidence for the specificity of this relation, as there was no difference in the relation between inhibitory control and PM in the verbal interference as compared to the standard condition. Thus, it is likely that the relation is language-specific, as better inhibition did not relate to better PM under conditions of verbal interference. After controlling for age, however, the relation between PM and verbal ability was weaker but still of a medium effect size.

The current study supports past research that has suggested that active refreshing and rehearsal processes are occurring during the delay period in young children and that these processes support later PM performance (Mahy \& Moses, 2011, 2015). This study builds on this work and demonstrates that verbal processes such as subvocal rehearsal may play an important role in maintaining a prospective intention even in very young children. Importantly, these results suggest that subvocal rehearsal may support children's cognitive performance in a number of different domains. Further, our results suggest that verbal interference during the delay period had a less negative impact on PM for children with superior verbal ability compared to children with lower verbal ability. Future work should continue to examine the mechanisms through which children maintain their PM intentions as well as the underlying processes that support PM intention maintenance.

\section{Acknowledgments}

The authors wish to acknowledge Cicelia Arsenault, Hannah Bild-Enkin, Stephanie Bryan, Becca Koessler, Mary Makowski, Jayati Ramakrishnan, and Ryan Slight for assistance with data collection. Preparation of this manuscript was supported by an NSERC Discovery Grant to CEVM.

\section{References}

Al-Namlah, A. S., Fernyhough, C., \& Meins, E. (2006). Sociocultural influences on the development of verbal mediation: Private speech and phonological recoding in Saudi Arabian and British samples. Developmental Psychology, 42, 117-131.

Al-Namlah, A. S., Meins, E., \& Fernyhough, C. (2012). Self-regulatory private speech relates to children's recall and organization of autobiographical memories. Early Childhood Research Quarterly, 27, 441-446.

Baddeley, A. (1992). Working memory. Science, 255, 556-559.

Barrouillet, P., Bernardin, S., \& Camos, V. (2004). Time constraints and resource sharing in adults' working memory spans. Journal of Experimental Psychology, General, 133, 83-100.

Brandimonte, M. A., Ferrante, D., Feresin, C., \& Delbello, R. (2001). Dissociating prospective memory from vigilance processes? Psicológica: Revista De Metodología Y Psicología Experimental, 22, 97-114.

Carlson, S. M., \& Moses, L. J. (2001). Individual differences in inhibitory control and children's theory of mind. Child Development, $1032-1053$.

Carlson, S. M. (2005). Developmentally sensitive measures of executive function in preschool children. Developmental Neuropsychology, 28, 595-616.

Cowan, N. (1995). Attention and memory: An integrated framework. New York, NY: Oxford University Press.

Cowan, N. (2005). Working memory capacity. Hove, East Sussex, UK: Psychology Press.

Dunn, D. M., \& Dunn, L. M. (2007). Peabody picture vocabulary test: manual. Pearson.

Espy, K. A. (1997). The Shape School: Assessing executive function in preschool children. Developmental Neuropsychology, 13, 495-499. 
Fernyhough, C., \& Fradley, E. (2005). Private speech on an executive task: Relations with task difficulty and task performance. Cognitive Development, 20, $103-120$.

Hongwanishkul, D., Happaney, K. R., Lee, W. S., \& Zelazo, P. D. (2005). Assessment of hot and cool executive function in young children: Age-related changes and individual differences. Developmental Neuropsychology, 28, 617-644.

Jacques, S., \& Marcovitch, S. (2010). Development of executive function across the lifespan. In W. F. Overton (Ed.), Cognition, biology, and methods. Volume 1 of The handbook of life-span development (pp. 431-466). Hoboken, NJ: Wiley [Editor-in-Chief: R. M. Lerner].

Kliegel, M., \& Jäger, T. (2007). The effects of age and cue-action reminders on event-based prospective memory performance in preschoolers. Cognitive Development, 22, 33-46.

Kvavilashvili, L., Messer, D. J., \& Ebdon, P. (2001). Prospective memory in children: The effects of age and task interruption. Developmental Psychology, 37, 418-430.

Lidstone, J. S., Meins, E., \& Fernyhough, C. (2010). The roles of private speech and inner speech in planning during middle childhood: Evidence from a dual task paradigm. Journal of Experimental Child Psychology, 107, 438-451.

Lidstone, J., Meins, E., \& Fernyhough, C. (2011). Individual differences in children's private speech: Consistency across tasks, timepoints and contexts. Cognitive Development, 26, 203-213.

Müller, U., \& Kerns, K. (2015). The development of executive function. In L. S. Liben, U. Müller, \& R. M. Lerner (Eds.), Handbook of child of psychology and developmental science (vol. 2: Cognitive processes) (7th ed., vol. 2: Cognitive processes, pp. 571-623). Hoboken, NJ: Wiley.

Müller, U., Zelazo, P. D., Hood, S., Leone, T., \& Rohrer, L. (2004). Interference control in a new rule use task: Age-related changes, labeling and attention. Child Development, 75, 1594-1609.

Müller, U., Jacques, S., Brocki, K., \& Zelazo, P. D. (2009). The executive functions of language in preschool children. In A. Winsler, C. Fernyhough, \& N. Montero (Eds.), Private speech executive functioning, and the development of verbal self-regulation (pp. 53-68). Cambridge: Cambridge University Press.

Mahy, C. E. V., \& Moses, L. J. (2011). Executive functioning and prospective memory in young children. Cognitive Development, $26,269-281$.

Mahy, C. E. V., \& Moses, L. J. (2015). The effect of retention interval task difficulty on young children's prospective memory: Testing the intention monitoring hypothesis. Journal of Cognition and Development, 16, 742-758.

Mahy, C. E. V., \& Munakata, Y. (2015). Transitions in executive function: Insights from developmental parallels between prospective memory and cognitive flexibility. Child Development Perspectives, 9, 128-132.

Mahy, C. E. V., Moses, L. J., \& Kliegel, M. (2014). The development of prospective memory in children: An executive framework. Developmental Review, 34 $305-326$.

Ponitz, C. C., McClelland, M. M., Matthews, J. S., \& Morrison, F. J. (2009). A structured observation of behavioral self-regulation and its contribution to kindergarten outcomes. Developmental Psychology, 45, 605-619.

Vygotsky, L. S. (1986). Thought and language (A Kozulin, Trans.). Cambridge, MA: MIT Press [Original work published 1934].

Wechsler, D. (1974). Manual for the Wechsler intelligence scale for children-revised. New York: Psychological Corporation.

Wellman, H. M., \& Liu, D. (2004). Scaling of theory-of-mind tasks. Child Development, 75, 523-541.

Wellman, H. M., Cross, D., \& Watson, J. (2001). Meta-analysis of theory-of-mind development: The truth about false belief. Child Development, 72, 655-684.

Winsler, A., \& Naglieri, J. (2003). Overt and covert verbal problem-solving strategies: Developmental trends in use, awareness, and relations with task performance in children aged 5 to 17 . Child Development, 74, 659-678.

Winsler, A., de Leon, J. R., Wallace, B. A., Carlton, M. P., \& Willson-Quayle, A. (2003). Private speech in preschool children: Developmental stability and change, across-task consistency, and relations with classroom behaviour. Journal of Child Language, 30, $583-608$.

Zelazo, P. D. (2004). The development of conscious control in childhood. Trends in Cognitive Sciences, 8, 12-17. 